\title{
Development of a Smoke Transport Model to Enhance the Certification Process for Cargo Bay Smoke Detection Systems
}

\author{
Stefan P. Domino, Paul E. DesJardin, and Jill Suo-Anttila \\ Sandia National Laboratories* \\ PO Box 5800 MS 0836 \\ Albuquerque, New Mexico 87185-0836 \\ Telephone:505-284-6645, Email: jmsuoan@sandia.gov
}

\begin{abstract}
Current regulations require that aircraft cargo compartment smoke detectors alarm within one minute of the start of a fire and at a time before the fire has substantially decreased the structural integrity of the airplane. Presently in-flight and ground tests, which can be costly and time consuming, are required to demonstrate compliance with the regulations. A physics based Computational Fluid Dynamics (CFD) tool, which couples heat, mass and momentum transfer, has been developed to decrease the time and cost of the certification process by reducing the total number of both in-flight and ground experiments. The tool would provide information on smoke transport in cargo compartments under various conditions, therefore allowing optimal experiments to be designed. The CFD based smoke transport model will enhance the certification process by determining worst case locations for fires, optimum placement of fire detector sensors within the cargo compartment, and sensor alarm levels needed to achieve detection within the required certification time. The model is fast-running to allow for simulation of numerous fire scenarios in a short period of time. In addition, the model will be user friendly since it will potentially be used by airframers and airlines not expected to be experts in CFD. Following verfication of this CFD code, full-scale experiments will aid in the validation of the code and will gauge the reliability of using such a formulation to increase the efficiency of the aircraft fire detection system certification process by decreasing the total number of ground and flight experiments. This document includes a description of the CFD model and simulation studies developed to test the modeling strategy and numerical implementation of the CFD algorithm.
\end{abstract}

\footnotetext{
* Sandia National Laboratories is a multi-program laboratory operated by Sandia Corporation, a LockheedMartin Company, for the United States Department of Energy under Contract DE-AC04-94AL85000.
} 


\section{BACKGROUND}

Current regulations require that aircraft detectors within the cargo compartment of commercial airplanes provide a visual indication to the flight crew within one minute of the start of a fire. This time-to-detection time is based on a desire to detect a fire when small and at a state where temperatures are significantly below the temperature at which the structural integrity of the airplane is compromised. In-flight and ground tests are required to demonstrate compliance with these regulations. The objective of the FAA Smoke Transport Project is to develop a fast-running Computational Fluid Dynamics (CFD) based smoke transport model to assist in the certification of smoke detection systems in aircraft storage compartments. The model is to be suitable for interpreting flight test data and to be used in place of a number of flight tests that would be required during the certification process. Organizations in the user community include the FAA, airframers and airlines. The users are not expected to be experts in CFD.

\section{INTRODUCTION}

The essential features of the problem to be addressed include smoke transport in extensively packed (including many small regions between objects), ventilated compartments having comparably cold walls with potentially considerable curvature. A typical aircraft cargo compartment is shown in Figure 1. Also shown in the schematic are diagnostics (thermocouples, smokemeters, and gas analyzers) used during FAA full-scale testing in the 707 cargo compartment. Venting and potential fire sources are such that the flow may be driven by both ventilation and buoyancy. The spectrum of relevant scenarios includes high intensity (fast-growing) fires, and low intensity (smoldering) fires.

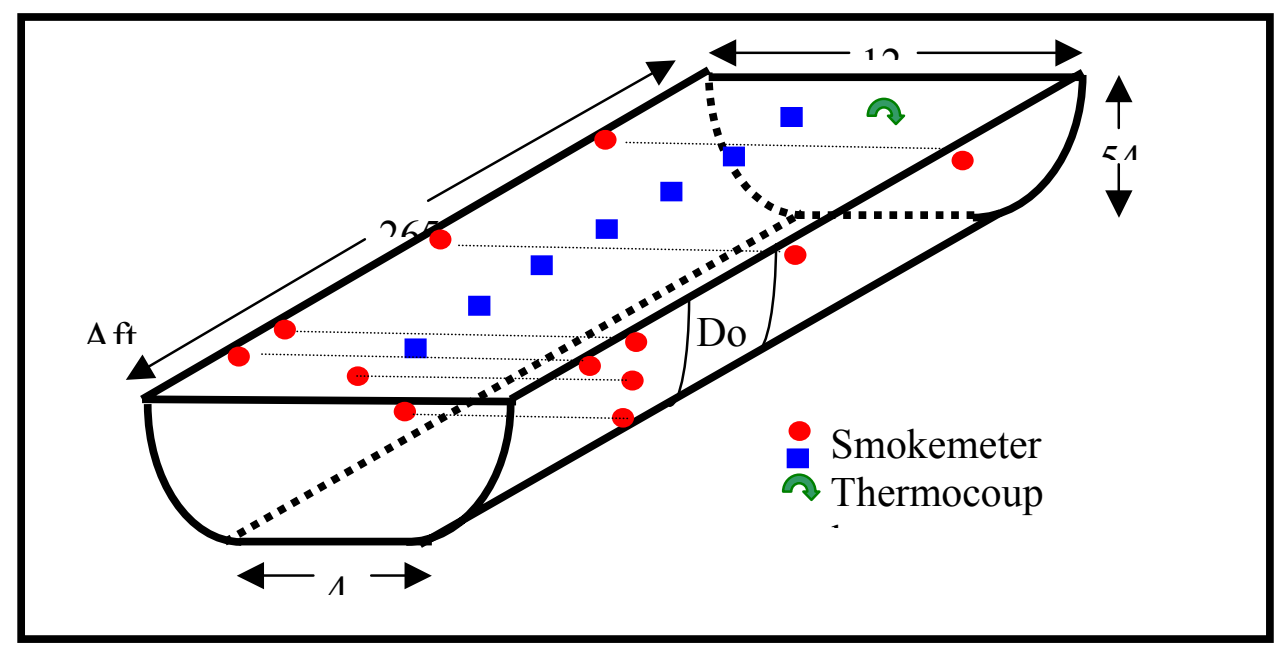

Figure 1. A typical aircraft cargo compartment geometry 
Based in part on observations of the flow characteristics of fire products during experiments at the FAA Technical Center, four classes of fire scenarios have been identified that may occur in aircraft cargo compartments (shown in Figure 2). Class 1 fires involve a buoyant plume that rises directly above a localized fire source, strikes the ceiling, and creates a ceiling jet flow. Class 2 fires are characterized by the plume attaching to a nearby wall before reaching the ceiling and creating a different flow pattern across the ceiling area. In both Class 1 and Class 2, the fire products fill the bay from the top down. In Class 3 fires, the smoke source is diffuse. Such scenarios are created by a source located within a large volume of cargo at the bottom of the compartment. Fire products from this class are apt to be relatively cool with respect to the ambient air by the time they reach the open region of the compartment and therefore may tend fill the compartment from the bottom. Class 4 fires would occur when the packages within the cargo compartment are containerized.

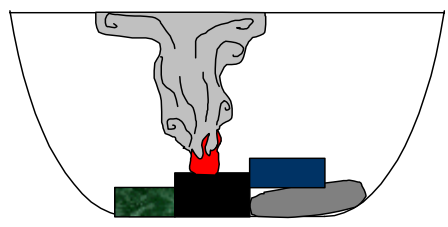

(1) Buoyant

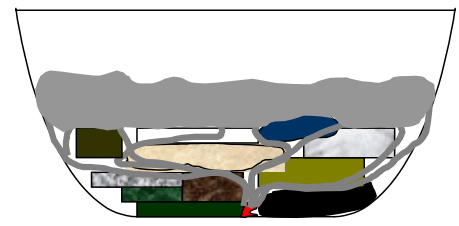

(3) Diffuse

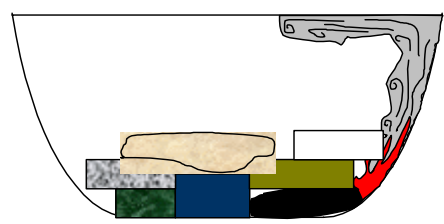

(2) Attached Flow

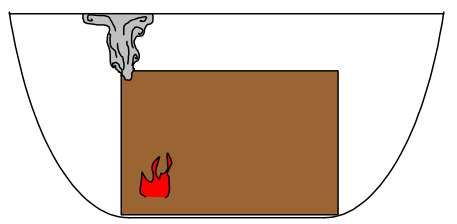

(4) Containerized

Figure 2. Three identified fire scenarios of interest

\section{SMOKE TRANSPORT MODELING}

Ideally, a physics based Computational Fluid Dynamics (CFD) tool, which couples heat, mass and momentum transfer, could be used to analyze the fire scenarios of interest. Use of the tool would result in an overall reduction in the time and cost of the certification process by providing a mechanism by which optimal in-flight and ground experiments are designed. The CFD model would serve to enhance the certification process by determining worst case locations for fires, optimum placement of fire detector sensors within the cargo compartment, and sensor alarm levels needed to achieve detection within the required certification time. 


\section{Fire Source Specification}

Although it is possible to include physical models that adequately describe the detailed chemical reactions germane to the fire process, such a simulation would likely exceed both the targeted simulation run-time and platform constraints. In fact, detailed multiple step kinetic devolatilization models for the materials common in airplane cargo compartments are not available. Therefore, the CFD simulator will not attempt to model the complex physical process of species devolatilization, chemical dependent heat release, and the chemical reaction interaction between high temperature free radicals. Rather, the CFD simulator utilizes experimentally time-resolved species and heat release data in lieu of simulating the complex physical phenomena associated with physical objects burning. Researchers at the FAA Technical Center developed a repeatable fire source to potentially be used in certification tests and an oxygen consumption fire calorimeter was used to characterize the source by measuring the release rate of heat, mass, and visible smoke. The test specimen, composed of combustible materials found in passenger luggage articles, was subjected to external heat fluxes up to $100 \mathrm{~kW} / \mathrm{m}^{2}$. Heat release rate due to gas phase combustion were determined by measuring oxygen depletion of the air drawn past the burning specimen into an exhaust duct where a laser system measures visible light obscuration of the smoke. Production of carbon dioxide, carbon monoxide, specimen mass loss, and heat release were measured during the tests with good repeatability. Figure 3 displays a typical time-resolved data set for experimentally measured heat, mass, and species release data that can be utilized within a CFD simulation.

The CFD simulator, therefore, numerically models the fire by the placement of volumetric mass and heat source terms as measured during the calorimeter experiments. The overall volumetric mass source term appears on the right hand side of the following equations: 1) continuity equation, 2) species transport equation (multiplied by the appropriate mass fraction of that particular species), and 3) the momentum equations in the form of a momentum sink. The heat release rate appears on the right hand side of the sensible enthalpy equation. The placement of volumetric heat releases on the computational grid will model the buoyantly induced flow rather than the associated heat release due to both homogeneous and heterogeneous chemical reaction. 

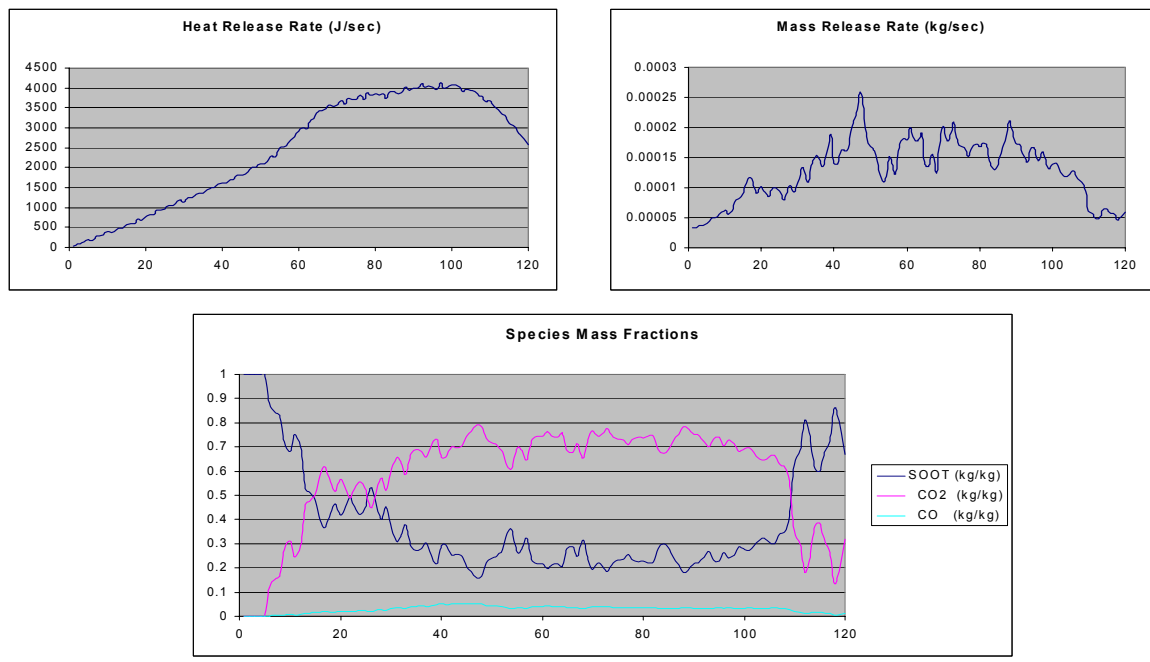

Figure 3. Time-resolved heat release, mass release, and species data

Although the technique of prescribing source terms is certainly not the preferred method for an entirely predictive CFD code, in this particular application where source terms are available through a detailed time-resolved experiment, it is the preferred method.

In addition to solving the time-mean equations describing the transport of momentum, equations describing the turbulent time-mean transport of germane species, e.g., CO, $\mathrm{CO}_{2}$, soot, etc., are computed and used for the calculation of point wise mixture properties such as molecular weight and heat capacity. A sensible enthalpy transport equation, including convection heat loss to the cargo walls, is solved to determine the temperature field using the mixture average heat capacity.

Upon preliminary testing of this CFD code, full-scale experiments will aid in the validation of the code and will gauge the reliability of using such a formulation to increase the efficiency of the aircraft fire detection system certification process by decreasing the total number of ground and flight experiments.

\section{CFD Model Formulation}

The formulation includes the description of the partial differential equation (PDE) set used to model the physics of the fire includes the Favre-Averaged, low- mach number representation of the Navier-Stokes equations and general transport equations for scalars such as species mixture fraction, sensible enthalpy, turbulent kinetic energy, and turbulent dissipation rate. A pressure-based method is used to obtain the pressure field by manipulation of the continuity and momentum equations to yield a pressure correction 
equation. The finite-volume method is used for the discretization of the partial differential equation set. The numerical scheme is cell centered, i.e., primitive variables and scalars are solved for at the control volume centers. A backward Euler method is employed and operator splitting is repaired via Picard iterations. For geometric flexibility, the equation set is transformed to a general non-orthogonal body-fitted coordinate system and solved in strongly conserved form.

Accurately modeling the complex physical phenomena associated with heterogeneous combustion often requires physical models that couple turbulent fluid flow, heat and mass transfer, radiant energy transfer, and chemical reaction. The appropriate physical governing transport equations, within integral form, are discretized and solved on a computational mesh. Unfortunately, the computational expense of solving the turbulent reacting system directly for all appropriate time and length scales frequently exceeds both the computational resources of the user and the desired cost-to-accuracy ratio. Therefore, models that are largely guided by reasonable engineering assumptions have been developed to decrease the associated computational expense in solving these types of problems while attempting to preserve all controlling physical phenomena.

In addition to the time-mean equations describing the transport of momentum, equations describing the turbulent time-mean transport of germane species, e.g., $\mathrm{CO}, \mathrm{CO}_{2}$, soot, etc., can be computed and used for the calculation of point wise mixture properties such as molecular weight and heat capacity.

The partial differential equations describing momentum, species, turbulent energy, turbulent dissipation, and sensible enthalpy transport are linearized and discretized using the finite-volume method [13]. The method of finite-volume discretization is a conservative approach even at low discretization resolution. The discrete continuity equation, which includes the appropriate discrete volumetric mass source term, is used to form the pressure correction equation [9].

The governing equations are solved iteratively using a segregated approach using a fully implicit scheme, which is first order accurate in time. Updating the matrix coefficients through each sweep captures the non-linearity inherent to the original PDE equation set. The linear system of equations for the momentum field, species, turbulent dissipation and production, and sensible enthalpy are solved using the strongly implicit method of Stone [14] while the pressure correction equation is solved via a preconditioned conjugate gradient method [15]. A time iteration is considered converged when the maximum residual of all individual linear equations is below a user-defined value.

\section{Clutter Modeling for Densely Packed Compartments}

In densely packed cargo compartments the CFD grid required to resolve small scale features, such as individual luggage items, would require extremely long simulation times. In order to maintain affordable computations, a subgrid scale model is developed to account for the effects of small scale cargo without requiring excessive grid resolution. The subgrid model is based on phase or spatial averaging techniques for which large scale flow features are resolved on a CFD grid while the effects of unresolved solid obstacles are modeled. 
However, for densely packed cargo compartments only the porous media limit needs to be considered. The transport equations for conservation of mass, species and energy do not change much from their time averaged form except with the addition of void fraction in the temporal and spatial derivative terms and can be expressed in terms of a general transport equation for the scalar. This methodology will be in modeling of the diffuse fire scenario.

\section{RESULTS}

A concerted effort between the FAA and Sandia National Laboratories is underway to validate the CFD model by comparing the simulation results to detailed experiments that measure species and smoke concentrations at various locations within the cargo compartment. Therefore, two simulations have been run to test the efficacy of the chosen modeling and numerical approach. The chosen problems are for the 707 forward cargo compartment, illustrated in Figure 1. The simulations were selected to demonstrate model performance in the buoyant plume and attached flow scenarios.

The total number of grid points represented in each cargo compartment fire simulation is 10,000. Adiabatic boundary conditions were assumed for the cargo bay walls, however, the generalized formulation includes the ability to model potential heat loss affects due to cool walls. Although the computer code allows for placement of air inlets and outlets, i.e., leakage rates, the vessel was assumed to be completely sealed and the turbulence models were in use.

\section{Buoyant Plume Simulation}

The first simulation was performed to assess model capabilities for the buoyant plume fire scenario. A constant heat and mass release rate of $2.25 \mathrm{KJ} / \mathrm{s}$ and $50 . \mathrm{e}-6 \mathrm{Kg} / \mathrm{s}$, respectively, was used. These boundary values correspond to a single instance of timeresolved experimental data for a flaming resin from a FAA cone calorimeter experiment. The given maximum heat and mass release rate used in this simulation was assumed to prevail over the entire simulation. The total simulation time, using a somewhat stringent convergence criteria, was approximately 2.5 hours.

Figure 4 illustrates a typically desired time resolution plot of temperature values at two different locations. The first location is directly over the fire source and the second location is on the centerline, 1.5 meters off the $3.365 \mathrm{~m}$ centerline distance. It is seen that the time-to-detection for the first instance of the fire to reach the top location is approximately 2 seconds. Analysis of the simulation results showed that the model captured the dominant physics for the buoyant plume case, including rise of the buoyant plume, contact with the ceiling, development of a ceiling jet, and filling of the compartment with smoke. 


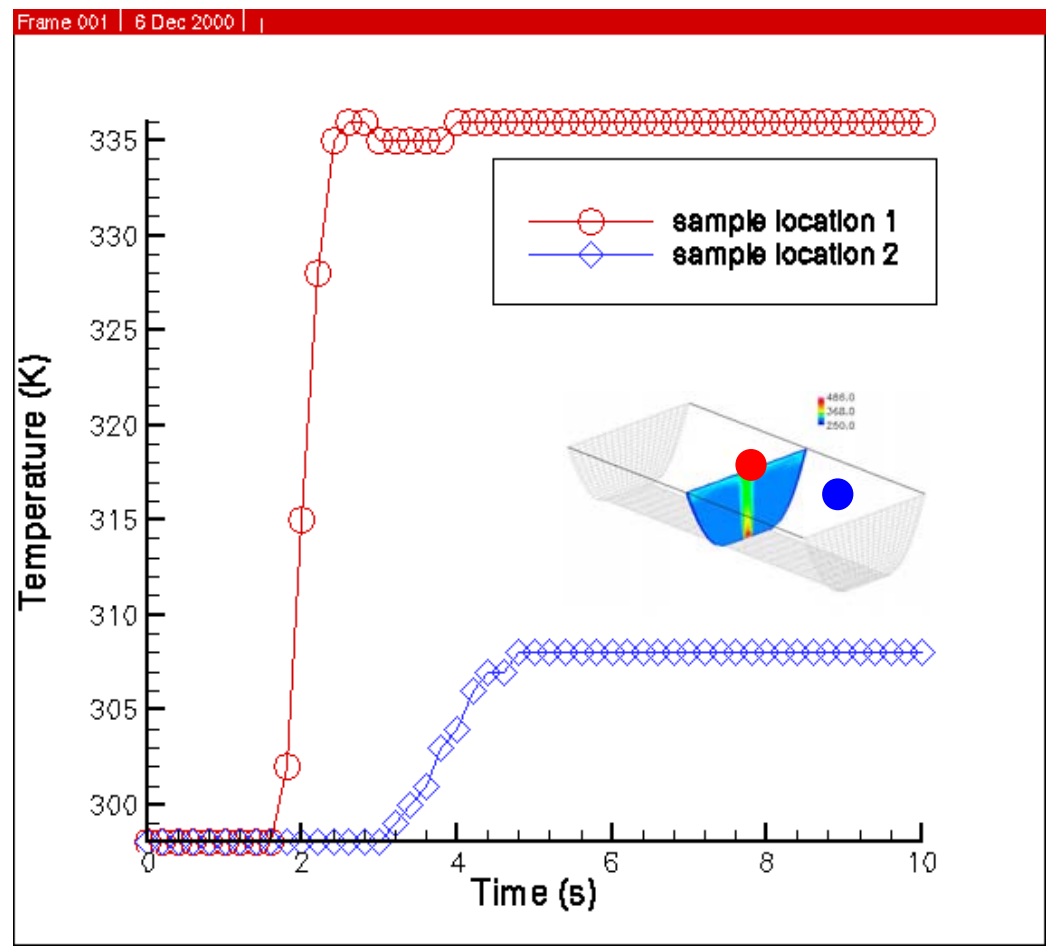

Figure 4. Buoyant Plume Scenario, temperature-history profile

\section{Attached Flow Simulation}

The second simulation was performed to show code capabilities for the attached flow fire scenario. The simulation includes the use of fully time-varying boundary conditions taken from a flaming case shown in Figure 3. At time zero, it is assumed that the heat and mass release rate is zero. Heat and mass release rates within an experimental point are determined by a linear interpolation between the scan interval.

The fire source was placed in the forward left corner of the bay and the simulation was allowed to run for 120 seconds of real experimental time. The maximum heat and mass release rates are approximately $4.0 \mathrm{KJ} / \mathrm{s}$ and $25 . \mathrm{e}-5 \mathrm{Kg} / \mathrm{s}$ respectively. The functionality of the split between $\mathrm{CO}$ and $\mathrm{CO} 2$ provides an interesting opportunity to observe the effect of a non-uniform product stream.

Figures 5 and 6 provide the detailed time-history of temperature, $\mathrm{CO} / \mathrm{CO}_{2}$ ratio, and soot mass fraction for the full two minute simulation. The probes shown are placed directly above the fire source and directly at the center of the bay as shown in the schematic in Figure 5. 


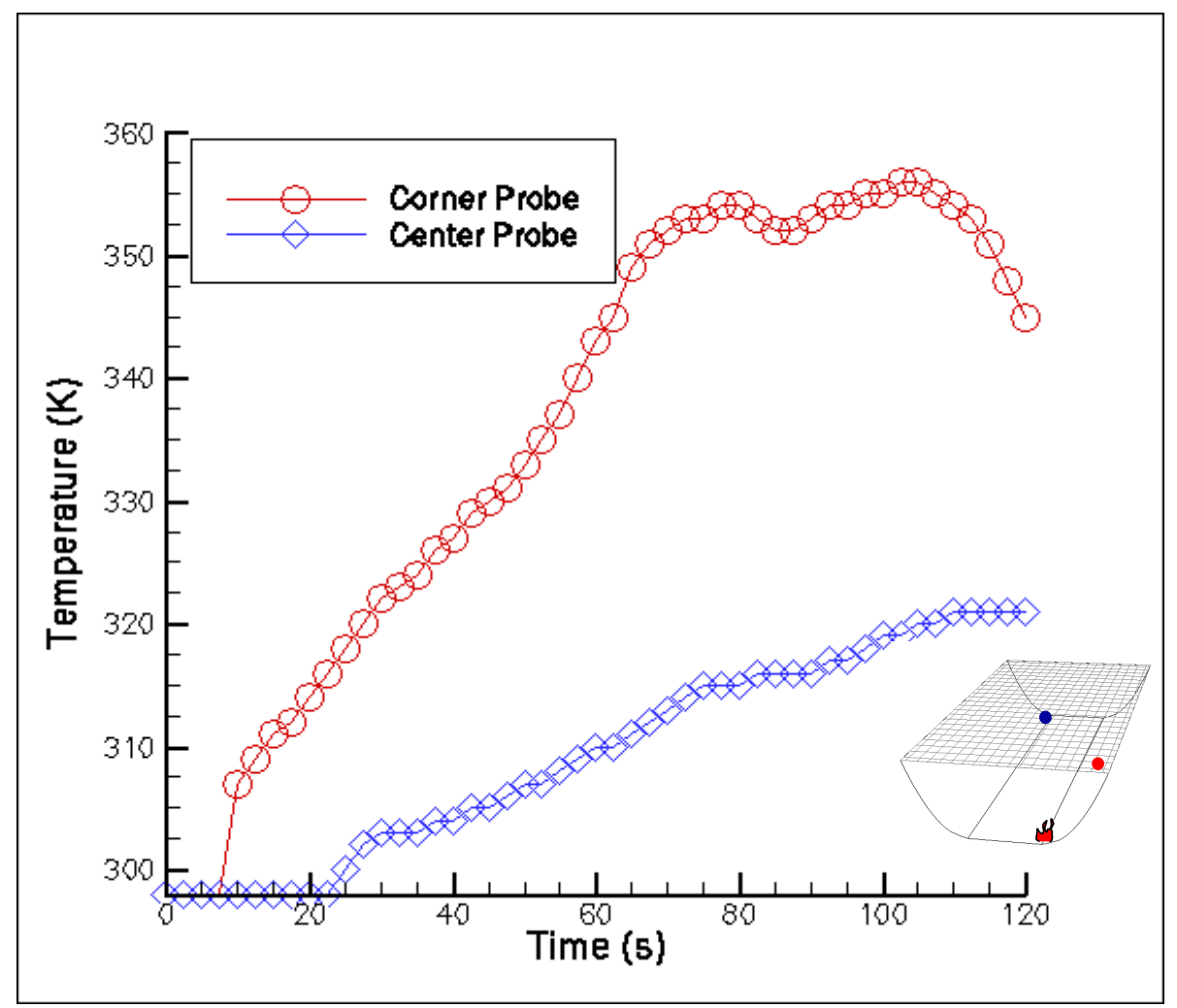

Figure 5. Attached Flow Scenario, temperature-history profile
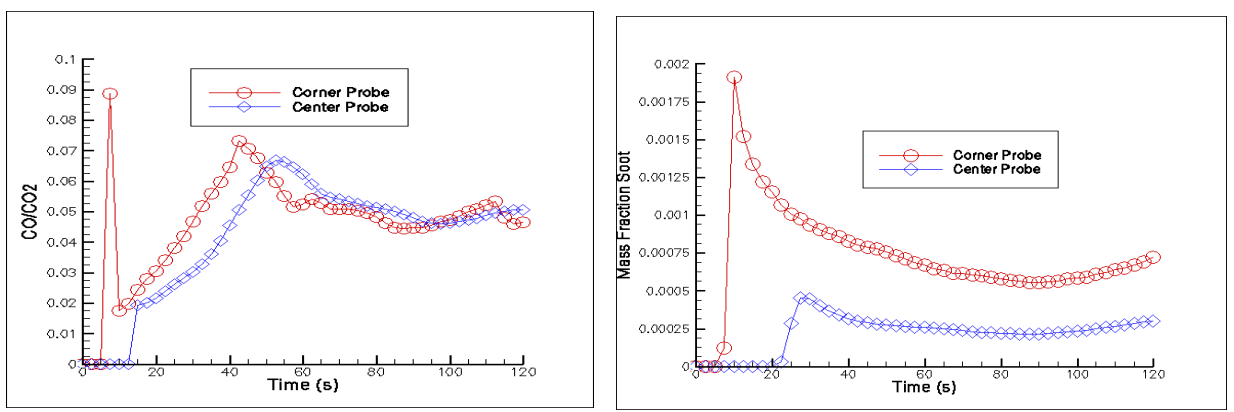

Figure 6. Attached Flow Scenario, $\mathrm{CO}, \mathrm{CO}_{2}$, and soot concentrations 
The most interesting aspect of the temperature plot resulting from the time-varying source case is the nature of the concave-up functionality of the temperature response at the overhead probe at approximately 85 seconds followed by a decrease in temperature. This behavior is due to the complex coupling between the thermal plume emanating from the corner source and the temperature wave that forms after the initial plume (ceiling plume) hits the far wall. This behavior is extremely apparent in the time-visualization of a "smoke" isosurface where it is observed that the smoke ceiling begins to drop just at the temperature at the probe increases.

The middle plot represents the ratio of $\mathrm{CO}$ to $\mathrm{CO}_{2}$ as a function of time at two probe locations. Note the highly variable split between the two species at the two different locations. This, for the first time, clearly demonstrates the advantage of computing multiple mass fraction transport equations when there are variable species release terms. Such a plot is anticipated to be highly valuable for use in the design of sensor threshold levels. The bottom plot is that of soot mass fraction and can also be used to guide sensor threshold levels. Again, the performance of the model for the attached flow class of problems is encouraging.

\section{CONCLUSION}

In this paper, a description of smoke transport code and example simulations of two fire scenarios were presented. The computer code draws upon detailed experimental data that are designed to provide time-varying boundary conditions for mass and heat sources.

The determination of the transient migration of species that are evolving from the fire source at different rates has been demonstrated. It is anticipated that the simulation tool can be extremely advantageous to the threshold design testing of $\mathrm{CO} / \mathrm{CO}_{2}$ sensors.

Results from the extended simulation of a two minute fire indicate that very complex flow patterns can arise due to the interaction of the evolving thermal plume and the ceiling jet. This complex interaction can drastically effect local temperatures. Moreover, much physical insight can be gained by the visualization of the smoke transport. Finally, it is noted that the total simulation time for a two minute fire, on a somewhat coarse mesh, is around 9 hours on a Sun Ultra 2.

Future efforts will focus on verification and validation of the CFD code for the application of interest. The validation process will primarily consist of comparison with experimental data from full-scale cargo compartment experiments conducted at the FAA Technical Center. These full-scale experiments will be used gauge the reliability of using the CFD-based smoke transport model to assist in the certification process of cargo compartment smoke detection systems. 


\section{ACKNOWLEDGEMENTS}

Sandia is a multi-program laboratory operated by Sandia Corporation, a Lockheed-Martin Company, for the United States Department of Energy under Contract DE-AC0494AL85000. This project was made possible by funding provided by the FAA. Appreciation is extended to David Blake of the FAA Technical Center for the graphics used in Figure 1. The authors would also like to acknowledge NASA Glenn Research Center and the FAA Technical Center for their support of this project.

\section{REFERENCES}

1) Bird, R. B., Steward, W. E. and Lightfoot, E. N., Transport Phenomena. John Wiley and Sons, New York, 1960.

2) Gatski, T. B., Grosch, C. E. and Rose, M. E., J. Comp. Phys., 82:298, 1989.

3) Favre, A. Problems of Hydrodynamics and Continuum Mechanics, Society of Indiustrial and Applied Mathematics, Philidelphia, 1969.

4) Bilger, R. W., Prog. Energy Combust. Sci., 1:88, 1976.

5) Launder, B. E. and Spalding, D. B., The Numerical Computation of Turbulent Flows, Computer Methods in Applied Mechanics and Engineering, 3:269, 1974.

6) DesJardin, P.E., Nelsen, J.M., Gritzo, L.A., Lopez, A.R., Suo-Anttila, J.M., Keyser, D.R., Ghee, T.A., Disimile, P.J. and Tucker, J.R., Proceedings of the Halon Options Technical Working Conference, 99-110, 2001.

7) Kuwahara, F., Kameyama, Y., Yamashita, S. and Nakayama, A., J. of Porous Meda, 1:47-55, 1998.

8) Ergun, S., Chem. Eng. Prog., 48:89-94, 1952.

9) Patankar, S. V., Numerical Heat Transfer and Fuid Flow, Taylor and Francis, New York, 1980.

10) Trefelthen, L. N. and Bau, D., Numerical Linear Algebra, SIAM, Philidelphia, 1997.

11) Rhie, C. M. and Chow, W. L., AIAA Journal, 21:1525-1532, 1983.

12) Parameswaran, S., Srinivasan, A. B. and Sun, R. L., N. H. T. Part A, 21:443, 1992.

13) Versteg, H. K. and Malalasekera, W., An Introduction to Computational Fluid Dynamics, Longman, London, 1999.

14) Stone, H., SIAM J. Numer. Anal., 5(3):530, 1968. 
15) Ferziger, J. H. and Peric, M., Computational Methods for Fluid Dynamics, Springer, New York, 1999.

16) Launder, B.E. and Spalding, D.B., Mathematical Models of Turbulence, Academic Press, London, 1972.

17) Mel, W. E., McGrattan, K. B. and Baum, H. R., Proc. Combust. Inst., 26:1523, 1996.

18) Tieszen, S. R., Nicolette, V. F., Gritzo, L. A.; Holen, J. K. and Moya, J. L., Vortical Structures in Pool Fires: Observation, Speculation, and Simulation, Sandia National Laboratories Technical Report No. SAND96-2607, Sandia National Laboratories, Albuquerque, New Mexico. 